Original Paper http://ajol.info/index.php/ijbcs http://indexmedicus.afro.who.int

\title{
Variation of essential oil composition of fruits of Zanthoxylum leprieurii Guill. et Perr (Rutaceae) from different sample locations and during their maturation period
}

\author{
Yoro TINE ${ }^{1,2^{*}}$, Alioune DIALLO ${ }^{1,2}$, William DIATTA ${ }^{3}$, Idrissa NDOYE ${ }^{1}$, Rokhya Sylla \\ GUÈYE ${ }^{1}$, Mamadou BALDÉ ${ }^{1}$, Adama DIÉDHIOU ${ }^{1}$, Djibril FALL ${ }^{1}$, Matar SECK ${ }^{1}$, \\ Jean-Marie DESJOBERT ${ }^{2}$, Jean COSTA ${ }^{2}$, Alassane WÉLÉ ${ }^{1}$ and Julien PAOLINI ${ }^{2}$ \\ ${ }^{1}$ Laboratoire de Chimie Organique et Thérapeutique, Faculté de Médecine, Pharmacie et Odontologie, \\ Université Cheikh Anta Diop, BP : 5005 Dakar-Fann, Sénégal. \\ ${ }^{2}$ Université de Corse, UMR CNRS 6134 SPE, Laboratoire de Chimie des Produits Naturels, Campus Grimaldi, \\ BP 52, F-20250 Corte, France. \\ ${ }^{3}$ Laboratoire de Pharmacognosie et de Botanique, Faculté de Médecine, Pharmacie et Odontologie, Université \\ Cheikh Anta Diop, BP : 5005 Dakar-Fann, Sénégal. \\ *Auteur correspondant ; E-mail: yoro.tine@ucad.edu.sn; Tél :00221774591945
}

\begin{abstract}
The fruits of Zanthoxylum leprieurii Guill. \& Perr. (Rutaceae) are traditionally used in Africa, particularly in Cameroon, as a spice and in the treatment of sickle cell anaemia. The objective of this study was to investigate the fruit essential oils from different sample locations and during their maturation period by using GC and GC-MS. Except for one sample collected in Aniak which consisted mainly of oxygenated monoterpenes, the volatile composition of $Z$. leprieurii fruits exhibited relative high amounts of monoterpene hydrocarbons (61.1-90.9\%) such as $(E)-\beta$-ocimene (13.4-75.2\%) and $\alpha$-pinene $(1.1-40.7 \%)$. The influence of the maturity stage on the chemical composition of the essential oils has resulted in an increase of oil yield and variations in the content of volatile compounds ( $\alpha$-pinene 13.3-75.6; $(E)-\beta$-ocimene 2.9-64.0\%).
\end{abstract}

Keywords: Senegal, Zanthoxylum leprieurii fruits, essential oil, chemical variability, stage of maturity and GC-MS.

\section{INTRODUCTION}

Zanthoxylum leprieurii Guil. \& Perr., also known as Fagara leprieurii Engl., is a tree about $24 \mathrm{~m}$ tall from the family Rutaceae, it is widespread throughout tropical Africa. In Cameroon, the dried fruits are traditionally used as spice in soups and its infusion is taken to treat sickle cell anaemia (Guetchueng et al., 2017; Tchinda et al., 2009). Some studies reported that fruit extracts exhibited antiplasmodial (Tchinda et al., 2009), cytotoxic (Choumessi et al., 2012; Ngoumfo et al., 2010) and antioxidant (Tchinda et al., 2009) activities. Previous phytochemical studies on the secondary metabolites of fruit extracts showed the presence of terpenes (Guetchueng et al., 2017), alkaloids (Ngoumfo et al., 2010; Tchinda et al., 2009) and coumarins (Tchinda et al., 2009). Moreover, some papers reported the chemical composition and biological 
activities of fruit essential oils from various geographical origins such as Nigeria and Cameroon. These studies showed chemical variability on the basis of amounts of hydrocarbon monoterpenes $((E)$ - $\beta$-ocimene, $\alpha$ pinene, $\beta$-pinene, terpinolene, limonene and $\delta$ 3 -carene), hydrocarbon sesquiterpene (caryophyllene), oxygenated monoterpenes $(\beta$ citronellol, citronellic acid and $\beta$-citronellal) (Dongmo et al., 2008; Ekundayo et al., 1986; Fogang et al., 2012; Gardini et al., 2009; Lamaty et al., 1989; Misra et al., 2013; Oyedeji et al., 2008; Reisch et al., 1985; Tatsadjieu et al., 2003).

Generally, the essential oil constituents depend on the internal and external agents influencing the plant such as genetic sequences, biotic, abioticand agricultural factors (Moniodis et al., 2018). In addition, seasonal variations (Matias et al., 2016; Moghaddam et al., 2015; Okoh et al., 2018), the development stage of the collected plant (Mehmood et al., 2019) and the method of preparing samples for analysis (Sefidkon et al., 2006) influence the yield and the different compound contents.

Thus, the aim of the present study was to characterize the chemical compositions of $Z$. leprieurii oil fruits from different sample locations and during their maturation period by using GC and GC-MS.

\section{MATERIALS AND METHODS \\ Plant material}

Twenty-seven samples of $Z$. leprieurii fruit pericarps (Figure 1) were collected between october 27 and 29, 2015 from seven localities of Senegal: Kabrousse (Kab, $12^{\circ} 21^{\prime} \mathrm{N}, 16^{\circ} 42^{\prime} \mathrm{O}$; eight samples), Emaye (Em, $12^{\circ} 26^{\prime} \mathrm{N}, 16^{\circ} 33^{\prime} \mathrm{O}$; two samples), Essyl (Es, $12^{\circ} 31^{\prime} \mathrm{N}, 16^{\circ} 25^{\prime} \mathrm{O}$; one sample), Colomba (Col, $12^{\circ} 46^{\prime} \mathrm{N}, \quad 16^{\circ} 14^{\prime} \mathrm{O}$; four samples), Thionck Essyl $\left(T h, 12^{\circ} 46^{\prime} \mathrm{N}, 16^{\circ} 28^{\prime} \mathrm{O}\right.$; four samples), Carapan (Cap, $12^{\circ} 51^{\prime} \mathrm{N}, 16^{\circ} 19^{\prime} \mathrm{O}$; five samples) and Aniak (An, 12 $344^{\prime} \mathrm{N}$, $16^{\circ} 07^{\prime} \mathrm{O}$; three samples). A sample corresponds to all the fruits harvested from the same tree.
For monitoring the evolution of the chemical composition of essential oil fruits during the maturation, the fruits were taken from a tree located in Colomba (Col.2) at different times (from september 1 to october 27, 2015). The plant material was identified by Dr William Diatta from the Department of botanical and pharmacognosy of University Cheikh Anta Diop of Dakar by comparison with the reference specimen deposited in the herbarium of this institution under number 1450.

\section{Essential oil isolation}

Plant material were air-dried for 14 days at room temperature. Samples were hydrodistilled (6 h) using a Clevenger-type apparatus according to the method recommended in the European Pharmacopoeia (Council of Europe, 1997). The yields of essential oils ( $\mathrm{w} / \mathrm{w}$, calculated on dry weight basis) are given in Table 1 and 2 . The essential oils were stored in amber glass bottles at a temperature of $4{ }^{\circ} \mathrm{C}$.

\section{Gas chromatography (GC) and gas chromatography-mass spectrometry (GC/MS) analysis}

Analyses were carried out using a Perkin-Elmer Autosystem XL GC apparatus (Walthon, MA, USA) equipped with dual flame ionisation detection (FID) system and fused-silica capillarycolumns, namely, Rtx-1 (polydimethylsiloxane) and Rtx-wax (polyethyleneglycol) $(60 \mathrm{~m} \times 0.22 \mathrm{~mm}$ i.d; film thickness $0.25 \mu \mathrm{m})$. The oven temperature was programmed from 60 to $230{ }^{\circ} \mathrm{C}$ at $2{ }^{\circ} \mathrm{C} / \mathrm{min}$ and then held isothermally at $230{ }^{\circ} \mathrm{C}$ for $35 \mathrm{~min}$ : hydrogen was employed as carrier gas (1 $\mathrm{mL} / \mathrm{min})$. The injector and detector temperatures were maintained at $280{ }^{\circ} \mathrm{C}$, and samples were injected $(0.2 \mu \mathrm{L}$ of pure oil) in the split mode (1:50). Retention indices (RI) of compounds were determined relative to the retention times of a series of n-alkanes (C5C30) by linear interpolationusing the Van den Dool and Kratz (1963) equation with the aid of software from Perkin-Elmer (Total Chrom navigator). The relative percentages of the oil 

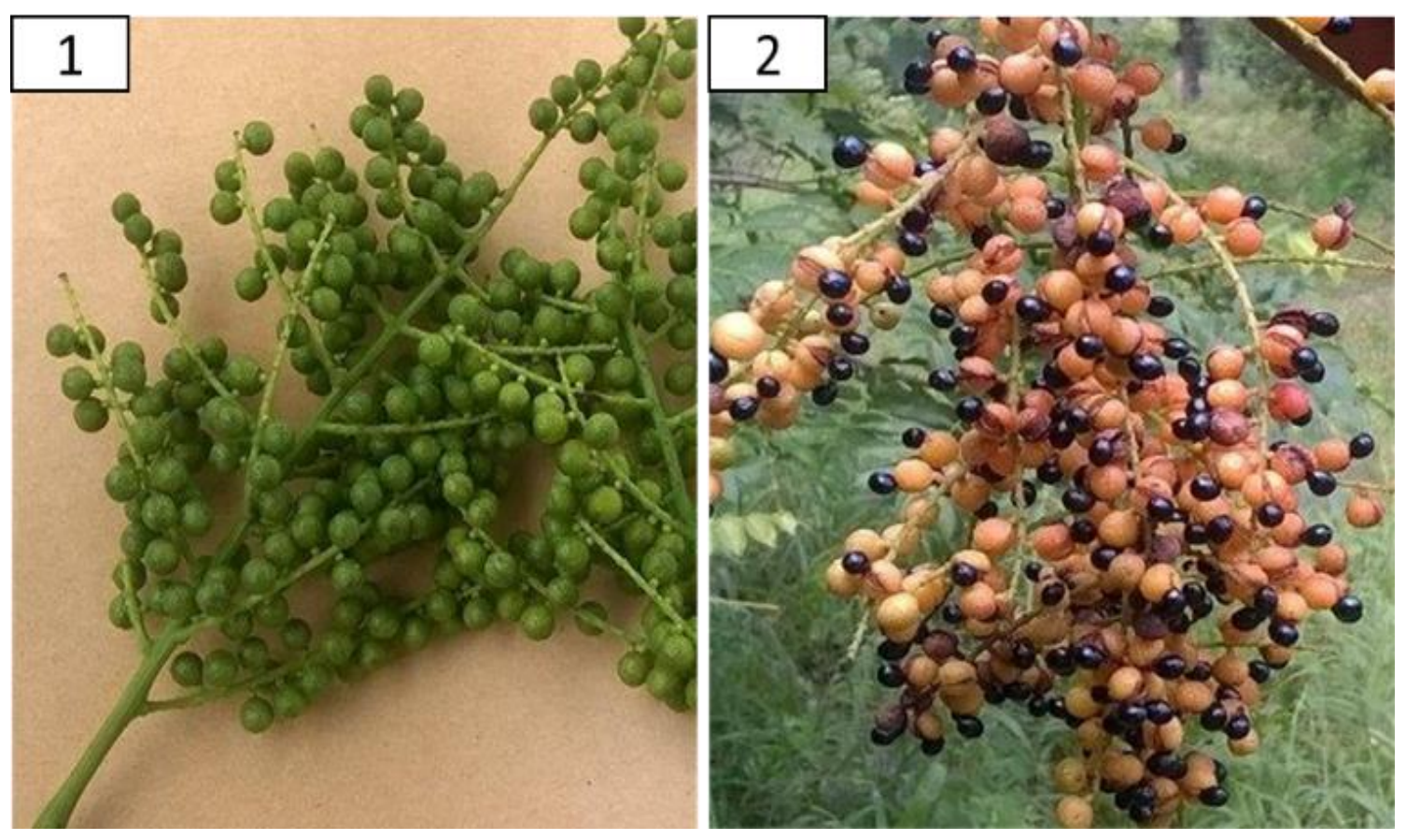

Figure 1 : Imature (1) and mature Fruits (2) of Z. leprieurii.

constituents were calculated from the GC peak areas, without application of correction factors. Samples were also analysed with a Perkin-Elmer Turbo mass detector (quadrupole) coupled to a PerkinElmerAutosystem $X L$, equipped with fusedsilica capillary columns Rtx-1 and Rtx-Wax. The oven temperature was programmed from 60 to $230{ }^{\circ} \mathrm{C}$ at $2{ }^{\circ} \mathrm{C} / \mathrm{min}$ and then held isothermally at $230{ }^{\circ} \mathrm{C}$ (35 min): hydrogen was employed as carrier gas $(1 \mathrm{~mL} / \mathrm{min})$. The following chromatographic conditions were employed: injection volume, $0.2 \mu \mathrm{L}$ of pure oil; injector temperature, $280{ }^{\circ} \mathrm{C}$; split, 1:80; ion source temperature, $150{ }^{\circ} \mathrm{C}$; ionisation energy, $70 \mathrm{eV}$; MS (EI) acquired over the mass range, 35-350 Da; scan rate, $1 \mathrm{~s}$.

Identification of the components was based on: (a) comparison of their GC retention indices (RI) on non-polar and polar columns, determined from the retention times of a series of n-alkanes with linear interpolation, with those of authentic compounds or literature data; (b) on computer matching with commercial mass spectral libraries (Adams and others, 2007; König et al., 2004; NIST, 2008) and comparison of spectra with those of our personal library; and (c) comparison of RI, MS and NMR spectral data of authentic compounds or literature data.

\section{RESULTS}

Chemical variability of fruit essential oils according to geographical origins of samples

We had undertaken a comparative study of the chemical composition of the fruits according to the biotopes (harvesting areas) in order to develop a possible production of essential oils from plants growing spontaneously. This study on different Senegalese biotopes aimed to highlight a possible specificity compared to studies in the literature. This work involved 27 samples of $Z$. leprieurii fruits taken from seven different stations (Kabrousse Kab, Emaye Em, Essyl Es, Colomba Col, Thionck Essyl Th, Caparan Cap 
and Aniak An). A sample corresponded to all the fruits harvested from the same tree.

Table 1 shows the chemical composition of each sample and gives the essential oil yields. These were calculated relatively to the mass of dry vegetable matter and are between 0.60 and $3.49 \%$.

The analysis of the fruit essential oils by GC-FID and GC/MS allowed the identification of 66 compounds accounting for 79.2 to $96.8 \%$ of the total oil weight. These oils had qualitatively similar chromatographic profiles, but their compositions were quantitatively very variable. They were all dominated by hydrocarbon monoterpenes (61.1-90.9\%) such as $(E)$ - $\beta$-ocimene $16(13.4-75.2 \%)$ and $\alpha$ pinene 2 (1.1-40.7\%). However, there was an exception, the sample An.2 was mainly composed of oxygenated monoterpenes. Samples collected at Kabrousse, Emaye, Caparan (except Cap.5), Essyl and Colomba showed almost the same proportions in $(E)-\beta$ ocimene $16(22.5-57.9 \%)$ and $\alpha$-pinene 2 (20.4-40.7\%), while those of Thionk essyl and Aniak were characterized by high levels of $(E)$ $\beta$-ocimene 16 (54.4-72.7\%) and low levels of $\alpha$-pinene $2(1.0-11.2 \%)$.

We also reported two atypical chemical compositions for two samples Cap.5 and An.2 The main components of Cap.5 are $\delta$-3-carene $10(17.1 \%), \alpha$-pinene 2 (14.9\%), terpinolene $20(14.5 \%)$ and (E)- $\beta$-Ocimene 16 (13.4\%) while those of An.2 were geranyl acetate 53 (51.6\%), geraniol $47(19.4 \%)$, cironellol 45 $(4.7 \%)$ and (E)- $\beta$-ocimene 16 (4.7\%). In addition, among these compounds, five had very low or no abundance in the other 25 samples: $\delta$-3-carene $10 \quad(0.0-0.1 \%)$, terpinolene $20(0.0-0.6 \%)$, geranyl acetate 53
$(0.0-3.2 \%)$, geraniol $47(0.0-3.6 \%)$ and cironellol $45(0.0-2.5 \%)$

\section{Evolution of the chemical composition of essential oils of fruits during their maturation period}

It should be emphasized that the valuation of an essential oil requires a judicious determination of the harvest period. In order to determine the impact of the fruit ripening process, we followed the evolution of the chemical composition of essential oil over a period of two months (September - October) for fruits always harvested on same tree Col.2 (Figure 1). First, we found that the yield of essential oil, expressed as a percentage (\%) relative to the dry matter, increases during fruit ripening. Indeed, the best yields (0.97 to $1.64 \%$ ) were obtained in October when the harvested fruits were yellow-orange in color. For immature fruits harvested in September; the yields are from 0.51 to $0.81 \%$ (Table 2).

The chemical composition of the essential oils was qualitatively identical throughout the ripening of the fruits. Essential oils were mainly characterized by hydrocarbon monoterpenes (78.5 to $86.2 \%$ ), with a high abundance of $\alpha$-pinene and $(E)$ - $\beta$-ocimene. The results and discussion of this study focused only on these two major constituents. However, we noted quantitative variations between the oil compositions of fruits from the first two harvests (September 1 and 8, 2015) characterized by $\alpha$-pinene rising to 75.6 and $57.9 \%$, respectively and those of the fruits of the following harvests (from September 22 to October 27, 2015) characterized by the combination of $\alpha$-pinene (13.3 to 32.2\%) with (E) $\beta$-ocimene (40.5 to $64.0 \%$ ) which was always the major compounds (Figure 2). 


\section{Y. TINE et al. / Int. J. Biol. Chem. Sci. 14(2): 626-637, 2020}

Table 1: Chemical variability of essential oils from $Z$. leprieurii fruits according to sample locations in Senegal.

\begin{tabular}{|c|c|c|c|c|c|c|c|c|c|c|c|c|c|c|c|c|c|c|c|c|c|c|c|c|c|c|c|c|c|c|c|}
\hline \multirow{3}{*}{$\mathrm{No}^{\mathrm{a}}$} & \multirow{3}{*}{ Compounds } & \multirow{3}{*}{$\mathbb{I R l a}^{b}$} & \multirow{3}{*}{$\mathrm{Rla}^{\mathrm{c}}$} & \multirow{3}{*}{$\mathrm{RIp}^{d}$} & \multicolumn{27}{|c|}{ Chemical variability of fruit essential oilse } \\
\hline & & & & & \multicolumn{8}{|c|}{ Kabrousse } & \multicolumn{2}{|c|}{ Emaye } & \multicolumn{5}{|c|}{ Caparan } & \multicolumn{4}{|c|}{ Colomba } & \multirow{2}{*}{\begin{tabular}{|c|}
$\begin{array}{c}\text { Ess } \\
\mathrm{yl}\end{array}$ \\
Es \\
\end{tabular}} & \multicolumn{3}{|c|}{ Aniak } & \multicolumn{4}{|c|}{ Thionck Essyl } \\
\hline & & & & & $\begin{array}{c}\text { Kab } \\
1\end{array}$ & $\begin{array}{c}\text { Kab } \\
2\end{array}$ & $\begin{array}{c}\text { Kab } \\
3\end{array}$ & $\begin{array}{c}\text { Kab } \\
4\end{array}$ & $\begin{array}{c}\text { Kab } \\
5\end{array}$ & $\begin{array}{c}\text { Kab } \\
6\end{array}$ & $\begin{array}{c}\text { Kab } \\
7\end{array}$ & $\begin{array}{c}\text { Kab } \\
8\end{array}$ & $\begin{array}{c}E m \\
1\end{array}$ & $\begin{array}{c}E m \\
2\end{array}$ & $\begin{array}{c}\text { Cap } \\
1\end{array}$ & $\begin{array}{c}\text { Cap } \\
2\end{array}$ & $\begin{array}{c}\text { Cap } \\
3\end{array}$ & $\begin{array}{c}\text { Cap } \\
4\end{array}$ & $\begin{array}{c}\text { Cap } \\
5\end{array}$ & $\begin{array}{c}\text { Col } \\
1\end{array}$ & $\begin{array}{c}\text { Col } \\
2\end{array}$ & $\begin{array}{c}\text { Col } \\
3\end{array}$ & $\begin{array}{c}\text { Col } \\
4\end{array}$ & & An 1 & $\begin{array}{c}\text { An } \\
2\end{array}$ & $\begin{array}{c}\text { An } \\
3\end{array}$ & \begin{tabular}{|c|} 
Th \\
1
\end{tabular} & $\begin{array}{c}\text { Th } \\
2 \\
\end{array}$ & $\begin{array}{c}\text { Th } \\
3 \\
\end{array}$ & $\begin{array}{c}\text { Th } \\
4 \\
\end{array}$ \\
\hline 1 & $a$-Thujene & 932 & 922 & 1023 & 0.1 & 0.1 & 0.3 & - & 0.1 & 0.1 & 0.1 & - & 0.3 & 0.2 & - & - & 0.1 & 0.1 & 0.1 & - & 0.3 & 0.1 & - & 0.1 & - & - & $\operatorname{tr}$ & - & - & 0.3 & 0.1 \\
\hline 2 & $\alpha$-Pinene & 936 & 931 & 1015 & 35.5 & 20.4 & 33.3 & 40.7 & 35.2 & 29.0 & 38.7 & 28.4 & 23.7 & 30.7 & 34.4 & 25.9 & 30.1 & 30.2 & 14.9 & 29.1 & 30.4 & 16.1 & 26.1 & 35.3 & 1.1 & 2.0 & 2.5 & 11.2 & 9.7 & 6.4 & 6.0 \\
\hline 3 & Camphene & 950 & 943 & 1066 & 0.5 & 0.2 & 0.6 & 0.8 & 0.4 & 0.4 & 0.5 & 0.3 & 0.3 & 0.3 & 0.3 & 0.3 & 0.3 & 0.3 & 0.1 & 0.3 & 0.4 & 0.2 & 0.3 & 0.4 & - & - & tr & 0.1 & 0.1 & 0.1 & 0.1 \\
\hline 4 & Thuja-2,4-(10)-diene & 946 & 946 & 1123 & 0.1 & 0.1 & 0.1 & 0.1 & 0.1 & 0.1 & 0.2 & 0.1 & - & 0.1 & 0.1 & - & 0.1 & 0.2 & - & 0.1 & - & 0.1 & 0.1 & 0.3 & - & - & - & - & - & - & - \\
\hline 5 & Sabinene & 973 & 964 & 1120 & 0.5 & 0.4 & 3.2 & 0.1 & 1.1 & 0.1 & 0.8 & 0.1 & 4.0 & 2.2 & 0.1 & 0.1 & 0.1 & 0.1 & 0.1 & 0.1 & 2.7 & 0.3 & 0.1 & 0.5 & - & - & 1.1 & 0.1 & 0.1 & 6.0 & 3.5 \\
\hline 6 & $\beta$-Pinene & 978 & 970 & 1108 & 0.8 & 0.4 & 1.2 & 1.0 & 0.9 & 0.5 & 0.9 & 0.6 & 0.8 & 0.7 & 0.6 & 0.4 & 0.5 & 0.5 & 0.2 & 0.5 & 0.9 & 0.3 & 0.4 & 0.7 & - & $\operatorname{tr}$ & 0.1 & 0.3 & 0.2 & 0.4 & 0.4 \\
\hline 7 & Myrcene & 987 & 979 & 1159 & 1.0 & 0.6 & 1.5 & 1.6 & 1.1 & 1.0 & 1.1 & 0.8 & 1.2 & 1.0 & 0.7 & 1.0 & 1.0 & 0.7 & 4.5 & 0.9 & 1.3 & 0.6 & 0.8 & 0.7 & 0.3 & 2.9 & 0.4 & 0.6 & 0.6 & 0.9 & 0.7 \\
\hline 8 & Octanal & 981 & 980 & 1290 & 0.1 & 0.1 & $\operatorname{tr}$ & $\operatorname{Tr}$ & 0.1 & $\operatorname{tr}$ & 0.1 & 0.1 & 0.2 & $\operatorname{tr}$ & - & - & - & - & $\operatorname{tr}$ & 0.1 & 0.2 & 0.1 & tr & $\operatorname{tr}$ & 0.1 & 0.2 & 0.1 & 0.1 & - & - & - \\
\hline 9 & [-2-Carene & 1000 & 996 & 1131 & - & - & - & - & - & - & - & - & - & - & - & - & - & - & 0.5 & - & - & - & - & - & - & - & - & - & - & - & - \\
\hline 10 & ఇ-3-Carene & 1010 & 1005 & 1147 & - & - & - & - & - & - & - & - & - & - & - & - & - & - & 17.1 & - & - & - & - & - & - & - & - & - & - & 0.1 & - \\
\hline 11 & q-Terpinene & 1013 & 1008 & 1178 & 0.1 & 0.6 & 0.5 & - & 0.1 & 0.3 & 0.2 & 0.2 & 0.5 & 0.2 & - & - & - & - & 0.6 & tr & 0.4 & - & - & 0.2 & 0.2 & 0.2 & 0.1 & - & - & 0.5 & 0.5 \\
\hline 12 & $p$-Cymene & 1015 & 1011 & 1268 & 0.3 & 0.1 & 0.4 & 0.2 & 0.5 & tr & 0.3 & 0.1 & 0.6 & 0.3 & 0.1 & - & 0.2 & 0.3 & 2.0 & 0.2 & 0.5 & - & - & 0.3 & 0.1 & 0.2 & 0.3 & - & - & 0.6 & 0.7 \\
\hline 13 & Limonene & 1025 & 1020 & 1201 & 0.6 & 0.3 & 0.7 & 0.9 & 0.7 & 0.5 & 0.6 & 0.6 & 0.6 & 0.4 & 0.4 & 0.4 & 0.5 & 0.4 & 0.2 & 0.5 & 0.6 & 0.2 & 0.6 & 0.5 & 0.1 & 0.2 & 0.2 & 0.3 & 0.2 & 0.2 & 0.3 \\
\hline 14 & 1,8-Cineole & 1024 & 1020 & 1209 & 1.5 & 1.6 & 7.6 & 0.4 & 1.9 & 0.6 & 1.2 & 0.4 & 0.6 & 0.3 & 0.2 & 0.2 & 0.5 & 0.1 & 2.5 & 0.3 & 0.5 & 0.4 & 0.3 & 0.7 & 0.1 & 0.1 & 0.2 & 0.2 & 0.2 & 0.6 & 0.3 \\
\hline 15 & (Z)- $\beta$-Ocimene & 1029 & 1024 & 1230 & 0.6 & 0.6 & 0.5 & 0.5 & 0.4 & 0.7 & 0.6 & 0.5 & 0.6 & - & 0.5 & 0.8 & 0.5 & 0.6 & 0.3 & 0.7 & 0.7 & 1.0 & 0.8 & 0.4 & 1.0 & 0.1 & 0.8 & 0.9 & 1.1 & 1.0 & 0.7 \\
\hline 16 & $(E)-\beta$-Ocimene & 1041 & 1034 & 1247 & 41.0 & 51.5 & 33.1 & 32.6 & 24.2 & 45.8 & 36.4 & 29.1 & 41.4 & 49.7 & 36.4 & 52.6 & 32.5 & 39.6 & 13.4 & 48.7 & 50.9 & 62.6 & 57.9 & 22.5 & 72.7 & 4.7 & 65.5 & 70.4 & 75.2 & 64.1 & 54.4 \\
\hline 17 & П-Terpinene & 1051 & 1047 & 1243 & 0.2 & 0.2 & 0.8 & 0.1 & 0.3 & 0.1 & 0.2 & 0.1 & 1.1 & 0.4 & 0.1 & - & 0.2 & tr & 0.1 & $\operatorname{tr}$ & 0.7 & 0.1 & 0.1 & 0.3 & - & - & 0.4 & - & - & 1.0 & 1.2 \\
\hline
\end{tabular}




\begin{tabular}{|c|c|c|c|c|c|c|c|c|c|c|c|c|c|c|c|c|c|c|c|c|c|c|c|c|c|c|c|c|c|c|c|}
\hline 18 & Octanol & 1063 & 1065 & 1531 & 0.2 & - & - & 0.1 & & 0.1 & 0.3 & - & 0.1 & - & $\operatorname{tr}$ & - & 0.1 & 0.2 & - & - & 0.1 & 0.1 & - & - & 0.2 & - & - & - & - & 0.1 & 0.2 \\
\hline 19 & $p$-Cymenene & 1075 & 1072 & 1432 & - & - & - & - & - & - & - & - & - & - & - & - & - & - & 1.1 & 0.1 & - & - & - & - & - & - & - & - & - & 0.1 & - \\
\hline 20 & Terpinolene & 1082 & 1078 & 1280 & - & - & - & - & - & - & - & - & - & - & - & - & - & - & 14.5 & - & 0.4 & - & - & - & - & - & - & - & - & 0.6 & - \\
\hline 21 & $\begin{array}{l}\text { 6-Methyl-3,5- } \\
\text { heptadien-2-one }\end{array}$ & & 1080 & 1581 & 0.2 & 0.6 & 0.2 & 0.1 & 0.2 & 0.3 & 0.3 & 0.3 & - & 0.2 & 0.1 & - & 0.2 & 0.2 & - & 0.3 & - & 0.5 & 0.3 & 0.2 & 0.2 & 0.1 & 0.2 & 0.3 & 0.2 & 0.1 & 0.2 \\
\hline 22 & Linalool & 1086 & 1081 & 1544 & 0.3 & 0.4 & 0.2 & 0.3 & 0.3 & 0.3 & 0.2 & 0.7 & - & 0.3 & - & 0.1 & 0.1 & 0.3 & - & 0.2 & $\operatorname{tr}$ & 0.2 & 0.1 & 0.4 & 0.4 & 0.9 & 0.3 & - & 0.2 & 0.3 & 0.2 \\
\hline 23 & Rosefurane & 1091 & 1083 & 1398 & 0.1 & 1.9 & 1.0 & 0.9 & 1.2 & 1.4 & 1.9 & 1.7 & 0.9 & 1.2 & 1.2 & 0.4 & 2.3 & 2.0 & - & 2.0 & - & 1.5 & 1.1 & 3.5 & 1 & 0.6 & 1 & 1.1 & 0.8 & 0.3 & 1.6 \\
\hline 24 & Nonanal & 1076 & 1083 & 1394 & 1.1 & 0.2 & $\operatorname{tr}$ & 0.2 & 0.1 & 0.2 & 0.1 & 0.1 & - & 0.1 & - & - & 0.2 & 0.2 & - & 0.1 & 0.1 & 0.1 & 0.1 & 0.2 & 0.3 & 0.1 & 0.1 & 0.2 & 0.1 & 0.1 & - \\
\hline 25 & Perrilene & 1090 & 1092 & 1416 & - & - & - & 0.1 & - & - & - & - & - & 0.2 & - & - & - & - & 0.1 & 0.1 & - & - & - & 0.1 & - & - & - & - & - & - & - \\
\hline 26 & I-Campholenal & 1105 & 1105 & 141 & 0.2 & 0.1 & 0.1 & 0.2 & 0.2 & 0.3 & 0.2 & 0.3 & 0.2 & 0.2 & 0.1 & 0.1 & 0.3 & 0.2 & - & 0.3 & - & - & 0.1 & - & 0.1 & - & - & - & - & - & 0.1 \\
\hline 27 & $\begin{array}{l}\text { p-Mentha-1,3,8- } \\
\text { triene }\end{array}$ & 1123 & 1122 & 1433 & 0.2 & 0.2 & 0.2 & 0.4 & 0.2 & 0.1 & 0.1 & 0.2 & 0.2 & 0.2 & 0.2 & 0.1 & 0.2 & 0.3 & - & 0.3 & 0.7 & 0.3 & 0.2 & 0.3 & 0.3 & - & 0.3 & 0.3 & 0.4 & 0.4 & 0.2 \\
\hline 28 & (E)-Ocimenoxide & 1125 & 1125 & 1482 & 0.2 & 0.3 & 0.2 & - & 0.4 & 0.3 & 0.3 & 0.3 & 0.3 & 0.4 & 0.4 & 0.3 & 0.6 & 0.3 & - & 0.5 & 0.1 & 0.3 & 0.4 & 0.2 & 1.1 & - & - & 0.5 & 0.7 & 0.4 & 0.3 \\
\hline 29 & Cis-Verbenol & 1132 & 1127 & 1655 & 0.3 & 0.3 & 0.2 & 0.3 & - & 0.7 & 0.7 & 0.6 & - & - & - & - & 0.7 & 0.3 & - & 0.5 & - & - & - & 0.4 & - & - & - & - & - & - & - \\
\hline 30 & Citronellal & 1129 & 1131 & 1479 & - & - & - & - & - & - & - & - & - & - & - & - & - & - & - & - & - & - & - & - & - & 3.0 & - & - & - & - & - \\
\hline 31 & Pinocarvone & 1137 & 1136 & 1558 & 0.1 & 0.1 & 0.1 & 0.1 & 0.1 & 0.1 & 0.2 & 0.2 & 0.1 & 0.1 & - & - & 0.1 & 0.1 & - & - & - & - & - & 0.2 & - & - & - & - & - & - & 0.1 \\
\hline 32 & $\begin{array}{l}p \text {-Mentha-1,5-dien- } \\
\text { 8-ol }\end{array}$ & 1127 & 1146 & 1714 & 0.4 & 0.4 & 0.4 & 0.2 & 0.3 & 0.2 & 0.3 & 0.4 & 0.2 & 0.2 & - & - & 0.1 & 0.4 & - & 0.2 & - & 0.2 & 0.2 & 0.4 & 0.1 & - & 0.1 & 0.2 & 0.1 & tr & 0.2 \\
\hline 33 & Borneol & 1150 & 1148 & 1698 & 0.2 & 0.2 & 0.2 & 0.2 & 0.2 & 0.1 & 0.2 & 0.2 & - & 0.2 & - & - & - & - & - & - & - & - & 0.1 & 0.2 & 0.1 & - & 0.2 & - & - & - & 0.1 \\
\hline 34 & Nonanol & 1149 & 1156 & 1635 & 0.2 & 0.7 & - & 0.6 & - & 0.2 & 0.2 & 0.4 & - & 0.3 & - & - & 0.4 & 0.6 & - & 0.1 & - & 0.4 & 0.2 & 0.1 & 0.7 & 0.3 & 0.5 & 0.3 & 0.3 & 0.3 & 0.3 \\
\hline 35 & Cryptone & 1160 & 1157 & 1667 & 0.1 & - & 0.1 & - & 0.2 & 0.1 & - & - & - & - & - & - & - & - & - & - & - & - & 0.1 & - & - & - & - & - & - & - & - \\
\hline 36 & Terpinen-4-ol & 1164 & 1161 & 1600 & 0.6 & 0.6 & 2.2 & 0.3 & 1.5 & 0.1 & 0.7 & 0.3 & 3.6 & 1.0 & - & - & - & - & - & - & 1.8 & 0.3 & 0.2 & 0.6 & 0.2 & - & 3.3 & - & - & 3.0 & 6.5 \\
\hline 37 & p-Cymen-8-ol & 1169 & 1170 & 1833 & - & - & - & - & - & $\operatorname{tr}$ & - & - & - & - & - & - & 0.1 & 0.2 & 7.0 & 0.1 & - & - & - & - & - & - & - & - & - & - & - \\
\hline 38 & Myrtenal & 1172 & 1172 & 1628 & 0.1 & 0.1 & 0.1 & 0.1 & 0.1 & 0.1 & 0.1 & 0.1 & 0.1 & 0.1 & - & - & 0.2 & - & - & 0.1 & - & - & 0.1 & 0.2 & 0.1 & - & - & 0.1 & - & - & 0.1 \\
\hline 39 & Myrtenol & 1178 & 1177 & 1789 & 0.1 & - & - & 0.1 & 0.1 & 0.2 & 0.1 & 0.1 & - & - & - & - & - & - & - & - & - & - & - & 0.1 & 2.4 & - & 1.8 & 0.1 & - & - & - \\
\hline 40 & [-Terpineol & 1176 & 1179 & 1700 & 0.8 & 0.9 & 3.1 & 0.6 & 1.9 & 0.2 & 0.7 & 0.7 & 0.5 & 0.2 & - & - & 0.3 & 0.2 & 0.9 & 0.1 & 0.4 & 0.4 & 0.2 & 0.9 & 0.3 & - & 0.6 & 0.2 & 0.1 & 0.4 & 0.9 \\
\hline 41 & Verbenone & 1183 & 1184 & 1707 & 0.2 & - & 0.3 & - & 0.2 & 0.3 & 0.3 & - & - & 0.1 & - & - & - & - & - & - & - & - & - & - & - & - & - & 0.3 & - & - & - \\
\hline 42 & Decanal & 1180 & 1185 & 1498 & 0.2 & 0.7 & - & 0.8 & 0.7 & 0.2 & 0.1 & 0.4 & 0.1 & - & - & - & 0.6 & 0.6 & 0.2 & 0.2 & 0.1 & 0.5 & 0.4 & 0.4 & 0.6 & 0.9 & 0.4 & 0.3 & 1.2 & 0.3 & 0.4 \\
\hline 43 & Octyl acetate & 1194 & 1198 & 1478 & 0.6 & - & 0.2 & 0.3 & 0.4 & 0.4 & 0.8 & 0.1 & - & - & - & - & - & 0.7 & - & 0.3 & - & 0.6 & - & - & 0.6 & 0.2 & - & - & 0.3 & 0.2 & 0.7 \\
\hline 44 & Cis-Carveol & 1210 & 1208 & 163 & 0.1 & 0.2 & 0.1 & 0.2 & 0.1 & 0.2 & - & 0.2 & - & - & - & - & - & 0.1 & - & - & - & - & - & 0.1 & - & - & - & - & - & - & - \\
\hline
\end{tabular}


Y. TINE et al. / Int. J. Biol. Chem. Sci. 14(2): 626-637, 2020

\begin{tabular}{|c|c|c|c|c|c|c|c|c|c|c|c|c|c|c|c|c|c|c|c|c|c|c|c|c|c|c|c|c|c|c|c|}
\hline 45 & Citronellol & 1213 & 1208 & 1764 & - & - & - & 1.1 & 0.4 & - & - & 2.5 & 0.5 & - & - & - & - & - & 0.6 & - & - & - & - & - & - & 4.7 & - & - & - & 0.3 & - \\
\hline 46 & Nerol & 1210 & 1211 & 1799 & 0.3 & 0.2 & tr & - & - & 0.2 & - & - & - & 0.1 & - & - & - & 0.1 & - & - & - & - & - & - & - & 0.3 & - & - & - & - & - \\
\hline 47 & Geraniol & 1235 & 1232 & 1844 & - & - & - & 0.5 & 0.3 & - & - & 0.7 & 0.9 & - & - & - & - & - & 3.6 & - & - & - & - & - & 0.4 & 19.4 & - & - & - & - & - \\
\hline 48 & Geranial & 1244 & 1244 & 1731 & - & - & - & - & - & - & - & 0.1 & - & - & - & - & - & - & - & - & - & - & - & 0.1 & 0.3 & 0.4 & 0.2 & - & - & - & - \\
\hline 49 & Decanol & 1259 & 1261 & 1765 & - & - & - & 0.5 & - & - & - & 0.1 & - & - & - & - & - & - & - & - & - & - & - & - & 0.4 & - & - & - & - & - & - \\
\hline 50 & Bornyl acetate & 1270 & 1269 & 1575 & 0.1 & - & 0.1 & - & 0.1 & - & - & 0.1 & - & - & - & - & - & - & - & - & - & - & - & - & - & - & - & - & - & - & - \\
\hline 51 & Citronellyl acetate & 1337 & 1331 & 1657 & 0.1 & - & - & 0.3 & 0.1 & tr & - & 0.7 & 0.1 & - & - & - & - & - & 0.1 & - & - & - & - & - & - & 1.1 & - & - & - & - & - \\
\hline 52 & Neryl acetate & 1342 & 1342 & 1725 & 0.2 & 0.1 & tr & 0.1 & 0.3 & 0.1 & 0.1 & 0.3 & 0.6 & 0.1 & - & 0.1 & - & 0.1 & 0.1 & - & 0.2 & 0.2 & - & - & 0.1 & - & - & - & - & 0.5 & 0.2 \\
\hline 53 & Geranyl acetate & 1362 & 1361 & 1752 & 0.9 & 0.3 & - & 1.1 & 0.5 & 0.2 & $\operatorname{tr}$ & 1.1 & 3.0 & 0.2 & - & 0.1 & - & 0.2 & 3.2 & - & 0.4 & 0.1 & - & - & 0.5 & 51.6 & - & - & - & 0.5 & 0.1 \\
\hline 54 & $\begin{array}{l}\text { 3,5-heptdienal, 2- } \\
\text { ethylidene-6-methyl }\end{array}$ & & 1387 & 2039 & 0.2 & - & 0.2 & 0.3 & 0.3 & 0.4 & - & 0.6 & 0.2 & - & - & - & - & 0.3 & - & - & - & - & 0.3 & - & - & 0.1 & - & - & 0.5 & 0.2 & 0.4 \\
\hline 55 & I-Caryophyllene & 1421 & 1424 & 1591 & 0.6 & 0.7 & 0.8 & 0.8 & 1.6 & 1.2 & 0.5 & 1.3 & 1.1 & 0.4 & 4.5 & 5.0 & 1.1 & 1.5 & 0.6 & 0.8 & 0.5 & 0.7 & 0.7 & 1.2 & 0.3 & 0.2 & 2.2 & 0.6 & 0.4 & 2.3 & 1.6 \\
\hline 56 & a-Humulene & 1455 & 1450 & 1660 & 0.2 & 0.2 & 0.2 & 0.2 & 0.4 & 0.3 & 0.2 & 0.4 & 0.3 & 0.1 & 1.0 & 1.2 & - & 0.4 & 0.1 & 0.2 & 0.1 & 0.2 & 0.2 & 0.4 & - & - & 0.5 & 0.2 & 0.1 & 0.5 & 0.4 \\
\hline 57 & $\beta$-Selinene & 1486 & 1483 & 1712 & - & - & - & - & - & - & - & 0.3 & - & - & - & 0.2 & - & - & - & - & - & 0.1 & - & 0.2 & - & - & - & - & - & - & 0.1 \\
\hline 58 & I-Cadinene & 1520 & 1516 & 1752 & - & 0.1 & - & - & - & - & - & 0.2 & - & - & 0.1 & - & - & - & - & - & - & - & - & - & - & - & 0.1 & - & 0.1 & - & - \\
\hline 59 & (E)-Nerolidol & 1553 & 1546 & 2037 & 0.1 & 0.3 & 0.2 & 0.1 & 0.6 & 0.3 & - & 0.5 & 0.7 & 0.5 & 0.5 & 0.2 & - & 0.2 & - & 0.1 & 0.1 & 0.3 & 0.1 & 0.1 & - & 0.2 & 0.4 & - & 0.5 & - & 0.6 \\
\hline 60 & Germacrene B & 1552 & 1552 & 1828 & - & - & - & 0.2 & 0.5 & - & - & 0.2 & - & - & - & - & - & - & - & - & - & 0.2 & - & 0.2 & 0.5 & - & - & 0.3 & - & - & 0.4 \\
\hline 61 & Caryophyllene oxide & 1578 & 1576 & 1980 & 2.8 & 4.5 & 2.2 & 2.1 & 5.0 & 5.9 & 2.8 & 5.0 & 3.0 & 1.9 & 9.5 & 5.2 & 4.7 & 9.8 & 1.3 & 6.5 & 0.7 & 2.9 & 3.1 & 10.7 & 0.9 & 1.6 & 6.8 & 1.6 & 1.7 & 2.7 & 5.6 \\
\hline 62 & $\begin{array}{l}\text { Humulene epoxide } \\
\text { ॥ } \\
\text { Caryophylla- }\end{array}$ & 1602 & 1598 & 2044 & 0.6 & 1.0 & 0.8 & 0.4 & 1.0 & 0.9 & 0.6 & 1.0 & 0.6 & 0.3 & 1.2 & 0.7 & - & 1.4 & 0.2 & 1.0 & - & 0.5 & 0.5 & 2.2 & 0.2 & 0.3 & 1.1 & 0.4 & 0.3 & 0.4 & 0.8 \\
\hline 63 & $\begin{array}{l}\text { 4(14),8(15)-dien-5- } \\
\text { 띠 }\end{array}$ & & 1626 & 2285 & 0.1 & 0.3 & - & 0.1 & 0.2 & 0.2 & - & 0.4 & 0.1 & - & - & 0.1 & - & 0.3 & - & - & - & - & - & - & 0.1 & - & 0.2 & - & - & - & 0.1 \\
\hline 64 & $\begin{array}{l}\text { 14-Hydroxy-9-epi-E- } \\
\text { caryophyllene }\end{array}$ & & 1656 & 2316 & 0.3 & 0.4 & 0.1 & 0.1 & 0.4 & 0.6 & 0.2 & 0.4 & - & - & - & 0.5 & - & 0.9 & $\operatorname{tr}$ & 0.4 & - & - & 0.3 & 1.1 & - & - & 0.6 & - & - & 0.1 & 0.3 \\
\hline 65 & $(2 E, 6 E)$-Farnesol & & 1706 & 2336 & 0.4 & - & - & 0.5 & 0.4 & 0.1 & 0.1 & 0.4 & 1.0 & 0.3 & - & 0.3 & 0.3 & 0.1 & 0.2 & - & - & - & - & - & 0.7 & 0.2 & 0.2 & - & - & 0.2 & - \\
\hline 66 & $\begin{array}{l}(E-E) \text {-Farnesyl } \\
\text { acetate }\end{array}$ & 1822 & 1822 & 2260 & 0.2 & - & - & 0.1 & 0.1 & $\operatorname{Tr}$ & 0.1 & 0.4 & 0.2 & - & - & $\operatorname{tr}$ & - & - & $\operatorname{tr}$ & - & - & - & - & 0.1 & 0.1 & - & $\operatorname{tr}$ & - & - & 0.1 & - \\
\hline & $\begin{array}{l}\text { Hydrocarbon } \\
\text { Compounds }\end{array}$ & & & & 82.3 & 76.7 & 77.4 & 80.3 & 67.8 & 80.2 & 81.4 & 63.5 & 76.7 & 87.1 & 79.5 & 88.0 & 67.4 & 75.2 & 70.5 & 82.6 & 91.5 & 83.1 & 88.3 & 64.6 & 76.6 & 10.5 & 74.5 & 85.3 & 88.2 & 85.5 & 71.3 \\
\hline & $\begin{array}{l}\text { Oxygenated } \\
\text { Compounds }\end{array}$ & & & & 14.2 & 16.5 & 19.9 & 13.3 & 19.9 & 15.5 & 12.9 & 21.9 & 17.8 & 8.3 & 13.2 & 8.3 & 11.8 & 20.1 & 19.9 & 13.4 & 4.7 & 9.6 & 8.2 & 23.1 & 12.3 & 86.3 & 18.3 & 5.9 & 7.2 & 11.1 & 20.3 \\
\hline & Hydrocarbon Monoter & & & & 81.5 & 75.7 & 76.4 & 79.1 & 65.3 & 78.7 & 80.7 & 61.1 & 75.3 & 86.6 & 73.9 & 81.6 & 66.3 & 73.3 & 69.8 & 81.6 & 90.9 & 81.9 & 87.4 & 62.6 & 75.8 & 10.3 & 71.7 & 84.2 & 87.6 & 82.7 & 68.8 \\
\hline
\end{tabular}


Y. TINE et al. / Int. J. Biol. Chem. Sci. 14(2): 626-637, 2020

\section{Oxygenated Monoterpenes}

Hydrocarbon Sesquiterpenes

Oxygenated

Sesquiterpenes

Oxygenated non-terpenic compounds

Total identified

Yields (w/w vs dry material) $\begin{array}{lllllllllllllllllllllllllll}7.3 & 8.3 & 16.4 & 7.4 & 10.9 & 6.4 & 7.5 & 12.6 & 11.8 & 4.9 & 2.0 & 1.3 & 5.5 & 5.1 & 18.0 & 4.6 & 0.6 & 4.1 & 3.5 & 8.2 & 7.4 & 82.3 & 7.9 & 3.0 & 2.8 & 6.6 & 11.3\end{array}$

$\begin{array}{lllllllllllllllllllllllllll}0.8 & 1.0 & 1.0 & 1.2 & 2.5 & 1.5 & 0.7 & 2.4 & 1.4 & 0.5 & 5.6 & 6.4 & 1.1 & 1.9 & 0.7 & 1.0 & 3.4 & 1.2 & 0.9 & 2.0 & 0.8 & 0.2 & 2.8 & 1.1 & 0.6 & 2.8 & 2.5\end{array}$

$\begin{array}{llllllllllllllllllllllllllll}4.5 & 6.5 & 3.3 & 3.4 & 7.7 & 8.0 & 3.8 & 8.1 & 5.6 & 3.0 & 11.2 & 7.0 & 5.0 & 12.7 & 1.7 & 8.0 & 0.8 & 3.7 & 4.0 & 14.2 & 2.0 & 2.3 & 9.3 & 2.0 & 2.5 & 3.5 & 7.4\end{array}$

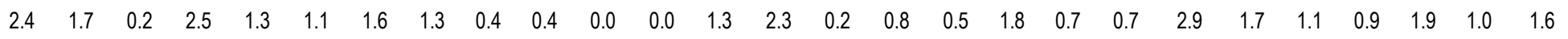

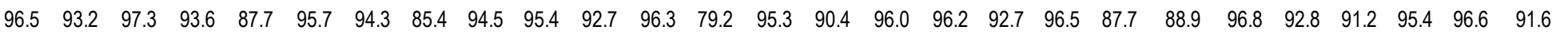

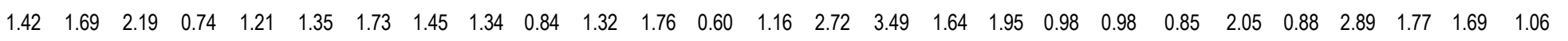

a Order of elution is given on apolar column (Rtx-1).

${ }^{\mathrm{b}}$ Retention indices of literature on the apolar column (IRla) (König et al., 2004).

c Retention indices on the apolar Rtx-1 column (Rla).

${ }^{d}$ Retention indices on the polar Rtx-Wax column (RIp).

e Localities of sampling.

tr: trace $(\%<0.05 \%)$

Table 2: Evolution of chemical composition of fruit essential oils of Z. leprieurii from Colomba Station (Col 2) during their maturation

\begin{tabular}{lcccccccc}
\hline Compounds (\%) & 01-sept & 08-sept & 22-sept & 29-sept & 06-oct & 13-oct & 20-oct & 27-oct \\
\hline$\alpha$-pinene & 75.6 & 57.9 & 26.8 & 17.1 & 13.3 & 18.0 & 32.2 & 30.5 \\
$(E)$ - $\beta$-ocimene & 2.9 & 8.9 & 40.5 & 58.2 & 64.0 & 64.0 & 53.1 & 55.7 \\
\hline Yields (\%) & 0.57 & 0.66 & 0.62 & 0.81 & 1.03 & 1.13 & 0.97 & 1.64 \\
\hline
\end{tabular}




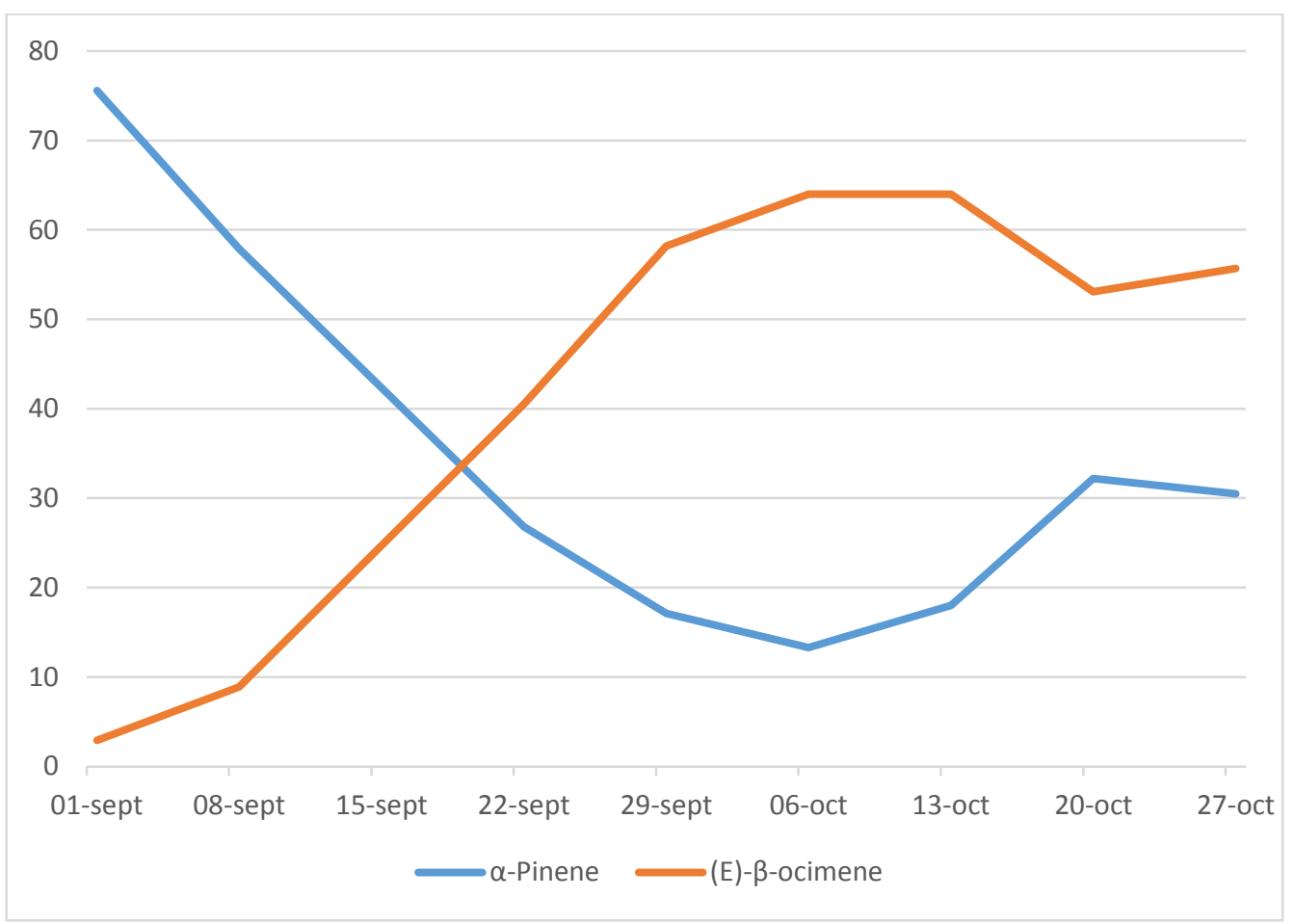

Figure 2: Evolution of the chemical composition of fruit essential oils of Z. leprieurii from Colomba Station (sample $\mathrm{Col} 2$ ) during their maturation period.

\section{DISCUSSION}

\section{Chemical variability of fruit essential oils} according to geographical origins of samples

The richness of fruit essential oils in (E)- $\beta$-ocimene has also been described in the literature, with relative percentages between $29.4 \%$ and $80.7 \%$ (Dongmo et al., 2008; Fogang et al., 2012; Lamaty et al., 1989; Misra et al., 2013; Tatsadjieu et al., 2003). On the other hand, an interesting content of terpinolene and $\delta$-3-carene in the fruit essential oils ( $26.0 \%$ and $14.5 \%$, respectively) has also been reported by Lamaty et al. (1989) from Cameroon (Lamaty et al., 1989). However, the chemotype, geranyl acetate/geraniol/cironellol/(E)- $\beta$-ocimene of the sample An.2, was well as the combination of $(E)$ - $\beta$-ocimene and $\alpha$-pinene in significant proportions $\quad(13.4-62.6 \%, \quad 14.9-40.7 \%$ respectively) has never been described in the literature to our knowledge.
Evolution of the chemical composition of essential oils of fruits during their maturation period

Despite the available reports on fennel essential oil composition, there are no previous references to the evaluation of the chemical composition of sweet fennel oil during fruit maturation. It is in this case not possible to compare our data with those of previous studies. Nevertheless, such studies were undertaken in other medicinal plants. In plants with different essential oil compositions in various ontogenetic periods such as coriander, essential oil composition is different. Schimitberger et al. (2018) reported $\delta$-3-carene (46.67\%) and $\alpha$-pinene $(14.98 \%)$ as the main compounds in immature fruit essential oil of Schinus terebinthifolia Raddi, while limonene $(37.49 \%)$ and $\delta$-3-carene $(19.98 \%)$ were the main compounds of mature fruit 
(Schimitberger et al., 2018). Telci et al. (2006) reported similar findings in coriander (Coriandrum sativum L.) with limonene contents varying from immature fruits (30\%) to full mature fruits (77\%) (Telci et al., 2006).

\section{Conclusion}

This study reported chemical variabilities of essentials oils of $Z$. leprieurii fruits from Senegal. According to the geographic origin of the populations, the composition of the fruit oils exhibited quantitative variations in the contents of hydrocarbon monoterpenes ((E)- $\beta$-ocimene and $\alpha$-pinene) except for a sample from Aniak which consists mainly of oxygenated monoterpenes. The influence of the maturity stage on the chemical composition of the essential oils has resulted in an increase of oil yield and variations in the content of volatile compounds. Therefore, this study could be useful for the valuation of the essential oil of the fruits of $Z$. leprieurii which requires a judicious determination of the harvest period.

\section{COMPETING INTERESTS}

The authors state that they have no conflict of interest.

\section{AUTHORS' CONTRIBUTIONS}

YT, JP, AW and JC conceived and coordinated the study. YT and JP designed, performed, and analyzed the experiments shown in the text. All authors reviewed the results and approved the final version of the manuscript.

\section{ACKNOWLEDGEMENTS}

We thank the Ministry of Higher Education and Scientific Research of Senegal, the Embassy of France in Senegal and the Territorial Authority of Corsica for their financial support.

\section{REFERENCES}

Adams RP. 2007. Identification of essential oil components by gas chromatography/mass spectrometry., Allured publishing corporation. Available from: https://www.cabdirect.org/cabdirect/abst ract/20083116584 (Consulté, le 31 Mai 2017).

Adesina SK. 1986. Further novel constituents of Zanthoxylum zanthoxyloides root and pericarp. J. Nat. Prod., 49(4): 715-716. DOI: https://doi.org/10.1021/np50046a035.

Choumessi AT, Loureiro R, Silva AM, Moreira AC, Pieme AC, Tazoacha A, Oliveira PJ, Penlap VB. 2012. Toxicity evaluation of some traditional African spices on breast cancer cells and isolated rat hepatic mitochondria. Food Chem Toxicol., 50(11): 4199-4208. DOI: https://doi.org/10.1016/j.fct.2012.08.008

Council of Europe. 1997. European Pharmacopoeia (3rd edn). Council of Europe: Strasbourg.

Dongmo PM, Tchoumbougnang F, Sonwa ET, Kenfack SM, Zollo PH, Menut C. 2008. Antioxidant and anti-inflammatory potential of essential oils of some Zanthoxylum (Rutaceae) of Cameroon. Int. J. Essen. Oil Ther., 2(2): 82-88.

Ekundayo O, Oguntimein B, Adeoye A. 1986. Volatile constituents of the essential oil of Zanthoxylum leprieurii. Fitoterapia, 57(4) : 267-269.

Fogang HPD, Tapondjou LA, Womeni HM, Quassinti L, Bramucci M, Vitali LA, Petrelli D, Lupidi G, Maggi F, Papa F, Vittori S, Barboni L. 2012. Characterization and biological activity of essential oils from fruits of Zanthoxylum xanthoxyloides Lam. and Z. leprieurii Guill. \& Perr., two culinary plants from Cameroon. Flavour Fragr. J., 27(2): 171-179. DOI: https://doi.org/10.1002/ffj.3083. 
Gardini F, Belletti N, Ndagijimana M, Guerzoni, ME, Tchoumbougnang $\mathrm{F}$, Zollo P HA, Micci C, Lanciotti R, Kamdem SLS. 2009. Composition of four essential oils obtained from plants from Cameroon, and their bactericidal and bacteriostatic activity against Listeria monocytogenes, Salmonella enteritidis and Staphylococcus aureus. Afr. J. Microbiol. Res., 3(5): 264-271.

Guetchueng ST, Nahar L, Ritchie KJ, Ismail Wansi JD, Evans AR, Sarker SD. 2017. Kaurane diterpenes from the fruits of Zanthoxylum leprieurii (Rutaceae). Rec. Nat. Prod., 11: 304-309.

König WA, Joulain D, Hochmuth D H. 2004. Terpenoids and Related Constituents of Essential Oils. Library of MassFinder, 2.

Lamaty G, Menut C, Bessiere JM, Aknin M. 1989. Aromatic plants of tropical Central Africa. II. A comparative study of the volatile constituents of Zanthoxylum leprieurii (Guill. et Perr.) Engl. and Zanthoxylum tessmannii Engl. leaves and fruit pericarps from the cameroon. Flavour Fragr. J., 4(4): 203-205. DOI: https://doi.org/10.1002/ffj.2730040411.

Matias EF, Alves EF, Silva MK, Carvalho VR, Figueredo FG, Ferreira JV, Coutinho HD, Silva, JM, Ribeiro-Filho J, Costa JG. 2016. Seasonal variation, chemical composition and biological activity of the essential oil of Cordia verbenacea DC (Boraginaceae) and the sabinene. Ind Crops Prod., 87: 45-53. DOI: https://doi.org/10.1016/j.indcrop.2016.04 .028 .

Mehmood T, Afzal A, Anwar F, Iqbal M, Afzal M, Qadir R. 2019. Variations in the Composition, Antibacterial and Haemolytic Activities of Peel Essential Oils from Unripe and Ripened Citrus limon (L.) Osbeck Fruit. J. Essent. OilBear. PL., 22(1): 159-168. DOI: https://doi.org/10.1080/0972060X.2019. 1588172
Misra LN, Wouatsa NV, Kumar S, Kumar RV, Tchoumbougnang F. 2013. Antibacterial, cytotoxic activities and chemical composition of fruits of two Cameroonian Zanthoxylum species. J. Ethnopharmacol., 148(1): 74-80. DOI: https://doi.org/10.1016/j.jep.2013.03.069

Moghaddam M, Miran SNK, Pirbalouti AG, Mehdizadeh L, Ghaderi Y. 2015. Variation in essential oil composition and antioxidant activity of cumin (Cuminum cyminum L.) fruits during stages of maturity. Ind Crops Prod., 70: 163-169. DOI:

https://doi.org/10.1016/j.indcrop.2015.03 .031 .

Moniodis J, Renton M, Jones CG, Barbour EL, Byrne M. Genetic and environmental parameters show associations with essential oil composition in West Australian sandalwood (Santalum spicatum). Aust. J. Bot., 66(1) :48-58. DOI: https://doi.org/10.1071/BT17116.

Ngoumfo RM, Jouda JB, Mouafo FT, Komguem J, Mbazoa CD, Shiao TC, Choudhary MI, Laatsch H, Legault J, Pichette A, Roy R. In vitro cytotoxic activity of isolated acridones alkaloids from Zanthoxylum leprieurii Guill. et Perr. Bioorg. Med. Chem., 18(10): 36013605.

DOI: https://doi.org/10.1016/j.bmc.2010.03.04 0.

NIST (National Institute of Standards and Technology). 2008. PC Version of the NIST/EPA/NIH Mass Spectra Library, [online] Available from: http://www.nist.gov/srd/nist1a.cfm (accessed on July 4, 2016).

Okoh SO, Okoh OO, Okoh AI. 2019. Seasonal variation of volatile oil composition and antioxidant property of aerial parts of Syzygium paniculatum Gaertn. grown in the Eastern Cape, South Africa. Nat Prod Res., 33(15): 2276-2280. DOI: https://doi.org/10.1080/14786419.2018.1 497032. 
Oyedeji AO, Lawal OA, Adeniyi BA, Alaka SA, Tetede E. 2008. Essential Oil Composition of Three Zanthoxylum Species. J. Essent. Oil Res., 20(1): 69-71. DOI:

https://doi.org/10.1080/10412905.2008.9 699425.

Reisch J, Adesina SK, Bergenthal D. 1985. Constituents of Zanthoxylum leprieurii fruit pericarps. Pharmazie, 40(11): 811812.

Schimitberger VMB, Pratti DL, Cavalcanti LC, Ramalho VF, Costa APF, Scherer R, Kuster RM, Ramos AC, Silva AG. 2018. Volatile compounds profile changes from unripe to ripe fruits of Brazilian pepper (Schinus terebinthifolia Raddi). Ind. Crops Prod., 119: 125-131. DOI: https://doi.org/10.1016/j.indcrop.2018.04 .011 .

Sefidkon F, Abbasi K, Khaniki GB. 2006. Influence of drying and extraction methods on yield and chemical composition of the essential oil of Satureja hortensis. Food Chem., 99(1): 19-23.

DOI: https://doi.org/10.1016/j.foodchem.2005. 07.026 .
Tatsadjieu LN, Essia Ngang JJ, Ngassoum MB, Etoa FX. Antibacterial and antifungal activity of Xylopia aethiopica, Monodora myristica, Zanthoxylum xanthoxyloides and Zanthoxylum leprieurii from Cameroon. Fitoterapia, 74(5): 469-472. DOI: https://doi.org/10.1016/S0367326X(03)00067-4.

Tchinda AT, Fuendjiep V, Sajjad A, Matchawe C, Wafo P, Khan S, Tane P, Choudhary MI. 2009. Bioactive compounds from the fruits of Zanthoxylum leprieurii. Pharmacologyonline, 1: 406-415.

Telci I, Bayram E, Avci B. 2006. Changes in yields, essential oil and linalool contents of coriandrumsativum varieties (var. vulgare Alef. and var. microcarpum DC.) harvested at different development stages. Eur. J. Hortic. Sci., 71(6): 267271. 